\title{
QUASICONFORMAL ANALOGUES OF A THEOREM OF SMIRNOV
}

\author{
BRUCE H. HANSON
}

\section{Introduction.}

Let $f$ be a conformal mapping of the unit disc $B^{2}=\{|x|<1\}$ onto the domain $D \subset \mathrm{C}$. Then there are a number of classical results which relate the size of $\left|f^{\prime}(x)\right|$ to the boundary behaviour of $f$. For example, we have the following well-known results due to Koebe and Smirnov respectively:

THEOREM 1. Assume $f$ is conformal on $B^{2}$. Then for any $x \in B^{2}$

$$
\frac{1}{2} \frac{d(f(x), \partial D)}{1-|x|} \leqq\left|f^{\prime}(x)\right| \leqq 4 \frac{d(f(x), \partial D)}{1-|x|} .
$$

(Here $d(y, A)$ is the distance from $y$ to $A$.)

THEOREM 2. Assume that $f$ is conformal on $B^{2}$ and that $D$ is a Jordan domain. Then $f^{\prime} \in H^{1}\left(B^{2}\right)$ if and only if $\partial D$ is rectifiable. Moreover, $f^{\prime} \in H^{1}\left(B^{2}\right)$ implies that $f$ is absolutely continuous on $\partial B^{2}$.

Astala and Gehring have shown that Koebe's Theorem (as well as a classical result of Hardy and Littlewood) has a rather direct analogue for quasiconformal mappings which remains true even in higher dimensions [AG1]. The main purpose of this paper is to show that there are quasiconformal analogues of Smirnov's Theorem as well.

\section{Notation.}

We use the expression $A \sim B$ to signify that the ratio $A / B$ is bounded away from 0 and $\infty$. The bounds on $A / B$ may depend on variables $x, y, \ldots$ In this case we write " $\sim$ " depends on $x, y, \ldots$ We use the expression $f: D \rightarrow E$ to signify that $f$ maps $D$ onto $E$. Let $B^{n}$ denote the unit ball in $R^{n}$. We assume for the remainder

Received January 21, 1993. 
of the paper that $f$ is a $K$-quasiconformal mapping of $B^{n}$ onto the domain $D \subset R^{n}$ with Jacobian $J_{f}$.

A direct analogue of Theorem 1 would involve replacing $\left|f^{\prime}(x)\right|$ by $\left(J_{f}(x)\right)^{\frac{1}{2}}$ in (1.1). This, of course, is not possible since $J_{f}$ need not be defined everywhere. In order to circumvent this difficulty, Astala and Gehring defined the following generalized version of $f^{\prime}(x)$ which has the advantage of being defined at every point in $B^{n}$ [AG1]:

$$
a_{f}(x)=\exp \left(\frac{1}{n m\left(B_{x}\right)} \int_{B_{x}} \log \left(J_{f}\right) d m_{n}\right)
$$

where $B_{x}=B(x,(1-|x|))$ is the open ball with center $x$ and radius $1-|x|$. Note that in the case where $n=2$ and $f$ is conformal, $a_{f}(x)=\left|f^{\prime}(x)\right|$. We also define the non-tangential maximal function of $a_{f}$ as follows:

$$
a_{f}^{*}(x)=\sup _{y \in \Gamma_{x}} a_{f}(y)
$$

where $x \in \partial B^{n}$ and $\Gamma_{x}=$ the interior of the closed convex hull of $\{x\} \cup B\left(0, \frac{1}{2}\right)$.

For $S \subset R^{n}$ let $\mathscr{H}_{\alpha}(S)$ denote the $\alpha$-dimensional Hausdorff measure of $S$. Abusing notation, we write $a_{f}^{p} \in H^{1}\left(B^{n}\right)$ (abbreviated $a_{f}^{p} \in H^{1}$ ) if $\sup _{r<1} \int_{S r} a_{f}^{p}(x) d \mathscr{H}_{n-1}(x)<\infty$, where $S_{r}=\{|x|=r\}$. We say that $\partial D \subset R^{n}$ is rectifiable if $\mathscr{H}_{n-1}(\partial D)<\infty$.

We next define John domains. Given an $\operatorname{arc} \gamma \subset R^{n}$ with endpoints $x_{0}$ and $x_{1}$, for each $x \in \gamma$ let $\gamma\left(x, x_{1}\right)$ denote the subarc of $\gamma$ with endpoints $x$ and $x_{1}$. For $b \geqq 1$ we define the b-carrot joining $x_{1}$ to $x_{0}$ by

$$
\operatorname{car}(\gamma, b)=\bigcup_{x \in \gamma}\left\{B\left(x, \frac{1}{b} d\left(\gamma\left(x, x_{1}\right)\right)\right)\right\},
$$

where $d(S)=$ diameter of $S$. A domain $G \subset R^{n}$ is called a $b$-John domain (with center $x_{0}$ ) if there exists $x_{0} \in G$ such that each $x_{1} \in G$ can be joined to $x_{0}$ by a $b$-carrot in $G$. $G$ is a John domain if $G$ is $b$-John for some $b<\infty$.

Closely related to John domains are uniform domains, which we next define. We say that a domain $G \subset R^{n}$ is uniform if there exist positive constants $a$ and $b$ such that each pair of points $x_{1}, x_{2} \in G$ can be joined by a rectifiable $\operatorname{arc} \gamma \subset G$ for which

$$
l(\gamma) \leqq a\left|x_{1}-x_{2}\right|
$$

and

$$
\min _{j=1,2} l\left(\gamma_{j}\right) \leqq b d(x, \partial G)
$$

for each $x \in \gamma$; here $l(\gamma)$ denotes the length of $\gamma$ and $\gamma_{1}, \gamma_{2}$ the components of $\gamma \backslash\{x\}$. Note that bounded uniform domains are John domains. 


\section{Statement of Results.}

We are now ready to state our results. We begin with two rather direct analogues of Theorem 2:

TheOREM A. Let $f: B^{2} \rightarrow D$ be K-quasiconformal, $D$ a Jordan domain. Then $a_{f} \in H^{1}$ if and only if $\partial D$ is rectifiable.

THEOREM B. Let $n \geqq 3$. Suppose that $D$ is a Jordan John domain in $R^{n}$ and that $f: B^{n} \rightarrow D$ is $K$-quasiconformal. Then $a_{f} \in H^{n-1}$ implies that $\partial D$ is rectifiable. Moreover, if we assume that $D$ is a uniform domain, then the converse holds, namely: if $\partial D$ is rectifiable, then $a_{f} \in H^{n-1}$.

These two theorems are proved in essentially the same way, but in higher dimensions the concept of rectifiability becomes more difficult to work with. This is the reason for the added restrictions on $D$ in Theorem B. Most likely the theorem remains true with less restrictive conditions placed on $D$. In fact, it is quite possible that no extra restrictions on $D$ are required in Theorem $B$ so that a direct generalization of Theorem $A$ holds in higher dimensions. In any case, the hypotheses on $D$ are always satisfied if $D$ is a quasiball, i.e. $D$ is the image of the unit ball under a mapping which is quasiconformal on all of $R^{n}$.

Note that neither of these results makes any mention of the absolute continuity of $f$ on $\partial B^{n}$ as was done in Theorem 2. In this regard the quasiconformal case is more complicated than the classical conformal case. In fact, when $n=2$ it is not true that $a_{f} \in H^{1}$ implies that $f$ is absolutely continuous on $\partial B^{2}$ as the following result shows:

Corollary C. For each $K>1$ there exists a K-quasiconformal $f: B^{2} \rightarrow B^{2}$ such that $a_{f} \in H^{1}$ but $f$ is singular on $\partial B^{2}$.

Corollary $\mathrm{C}$ is a simple consequence of Theorem $\mathrm{A}$ and the result of Beurling and Ahlfors [BA] that even in the disk a quasiconformal mapping can have singular boundary values.

Note that Corollary $\mathrm{C}$ is definitely not true in higher dimensions. In fact, if $n \geqq 3$ and $f: B^{n} \rightarrow B^{n}$ is quasiconformal, then $f$ is absolutely continuous on $\partial B^{n}$ without any assumptions on $a_{f}$ [G1, Theorem 1]. One could conjecture that for $n \geqq 3 a_{f} \in H^{n-1}$ implies that $f$ is absolutely continuous on $\partial B^{n}$. However, Juha Heinonen has recently constructed an example (for $n \geqq 3$ ) in which $\partial D$ is rectifiable, but $f$ is not absolutely continuous on $\partial B^{n}[\mathrm{H} 3]$. His example shows that this conjecture and the previous conjecture that Theorem $B$ holds without any extra hypotheses on $D$ cannot both be true.

In another related result [H1], Heinonen has shown (in the case $n \geqq 3$ and $n \neq 4$ ) that if $\partial D$ has tangents $\mathscr{H}_{n-1}-$ a.e., then $f$ is absolutely continuous on $\partial B$. 
In order to get $f$ absolutely continuous on $\partial B^{2}$ we need to assume more about $a_{f}$ :

ThEOREM D. Let $D$ be a Jordan domain in $R^{2}$ with $f: B^{2} \rightarrow D K$-quasiconformal. Then $a_{f}^{*} \in L^{1}\left(\partial B^{2}\right)$ implies that $f$ is absolutely continuous on $\partial B^{2}$.

Corollary $\mathrm{C}$ and Theorem $\mathrm{D}$ demonstrate a difference between the conformal theory and the quasiconformal theory in two dimensions. If $f$ is conformal, then $a_{f} \in H^{1}$ if and only if $a_{f}^{*} \in L^{1}$. On the other hand, if $f$ is quasiconformal, we only have the trivial implication $a_{f}^{*} \in L^{1}$ implies $a_{f} \in H^{1}$. One might conjecture that just as in the conformal case, $a_{f}^{*} \in L^{1}$ is equivalent with $f$ being absolutely continuous on $\partial B^{2}$, for quasiconformal $f$ 's. However, this is not true as the following result demonstrates:

THEOREM E. There exists $f: B^{2} \rightarrow B^{2}, K$-quasiconformal such that $f$ is absolutely continuous on $\partial B^{2}$ but $a_{f}^{*} \ddagger L^{1}\left(\partial B^{2}\right)$.

Our final result is similar to Theorem $D$. Note that Corollary C shows that this result is in some sense sharp.

TheEOREM F. Let $f: B^{2} \rightarrow D$ be K-quasiconformal, $D$ a Jordan domain, and $p>1$. Then $a_{f} \in H^{p}$ implies that $f$ is absolutely continuous on $\partial B^{2}$.

It is interesting to compare theorems $\mathrm{D}$ and $\mathrm{F}$ to the results in [C].

One should note that if $f$ is conformal, then $a_{f} \in H^{p}$ for some $p>1$ implies that $a_{f}^{*} \in L^{1}$, so in this case Therem $\mathrm{F}$ is an easy corollary of Theorem $\mathrm{D}$. I do not know whether the implication remains true in the quasiconformal case. It would be interesting to know if it does or not.

Theorem $\mathrm{F}$ is actually true with even weaker hypotheses on $a_{f}$. Let $\phi:[0, \infty) \rightarrow[0, \infty)$ be a homeomorphism satisfying

$$
\phi(x+y) \leqq C(\phi(x)+\phi(y))
$$

We say that $a_{f} \in H^{\phi}\left(B^{2}\right)$ if

$$
\sup _{r<1} \int_{S_{r}} \phi\left(a_{f}\right) d \mathscr{H}_{1}<\infty .
$$

Then a slight modification of the proof of Theorem $\mathrm{F}$ shows that we can replace the hypotheses that $a_{f} \in H^{p}\left(B^{2}\right)$ for some $p>1$ with the hypotheses that $a_{f} \in H^{\phi}\left(B^{2}\right)$ where $\phi$ satisfies (3.1) and

$$
\lim _{t \rightarrow \infty} \frac{\phi(t)}{t}=\infty
$$




\section{Tools.}

In this section we gather some of the results which we will need to prove the theorems stated in section 3 .

Our main tool will be the following analogue of Theorem 1 due to Astala and Gehring [AG1]:

TheOREM 3. Suppose $f: B^{n} \rightarrow D$ is K-quasiconformal. Then for any $x \in B^{n}$ we have

$$
\frac{1}{C} \frac{d(f(x), \partial D)}{1-|x|} \leqq a_{f}(x) \leqq C \frac{d(f(x), \partial D)}{1-|x|}
$$

where $C$ is a positive, finite constant which depends only on $K$ and $n$.

Before stating the next lemma, we need a definition. We say that $\left\{x_{1}, x_{2}, \ldots, x_{m}\right\} \subset F \subset S_{r}=\{|x|=r\} \subset B^{n}$ is a $\delta$-hyperbolic partition of $F$ if there exists $0<\delta<\infty$ such that the following two conditions are satisfied:

$$
\begin{gathered}
\min _{i \neq j}\left\{d\left(x_{i}, x_{j}\right)\right\} \geqq \delta(1-r) \\
\sup _{x \in F} \min _{1 \leqq i \leqq m} d\left(x, x_{i}\right) \leqq \frac{1}{\delta}(1-r) .
\end{gathered}
$$

We say that $\left\{x_{1}, x_{2}, \ldots, x_{m}\right\}$ is a hyperbolic partition of $F$ if $\left\{x_{1}, x_{2}, \ldots, x_{m}\right\}$ is a $\delta$-hyperbolic partition for some $\delta<\infty$. If $\left\{x_{1}, x_{2}, \ldots, x_{m}\right\}$ is a hyperbolic partition of $S_{r}$, we define

$$
P_{i}=\left\{x \in S_{r}: d\left(x, x_{i}\right)<d\left(x, x_{j}\right) j \neq i\right\} \quad i=1,2, \ldots, m .
$$

Then clearly the collection $\left\{P_{i}\right\}$ satisfies the following:

$$
\left\{P_{i}\right\} \text { is pairwise disjoint }
$$

$$
\begin{gathered}
\mathscr{H}_{n-1}\left(S_{r} \backslash\left\{\cup P_{i}\right\}\right)=0 \\
\mathscr{H}_{n-1}\left(P_{i}\right) \sim(1-r)^{n-1} \quad i=1,2, \ldots, m,
\end{gathered}
$$

where $\sim$ depends on $\delta$ and $n$. Note that for fixed $\delta$ small enough there will exist a $\delta$-hyperbolic partition of $S_{r}$ for each $r: 0<r<1$. For $n=2$ a natural choice is

$$
x_{j}=r e^{\frac{2 \pi j i}{m}} j=1, \ldots, m
$$

where $m \sim \frac{1}{1-r}$.

We are now ready to state 
LeMMA 1. Let $f: B^{n} \rightarrow D$ be K-quasiconformal and $\left\{x_{1}, \ldots, x_{m}\right\}$ a $\delta$-hyperbolic partition of $S_{r}, 0<r<1$. Then for $i=1, \ldots, m$

$$
\int_{P_{i}} a_{f}^{n-1} d \mathscr{H}_{n-1} \sim d\left(f\left(x_{i}\right), \partial D\right)^{n-1}
$$

and hence

$$
\int_{S_{r}} a_{f}^{n-1} d \mathscr{H}_{n-1} \sim \sum_{i=1}^{m} d\left(f\left(x_{i}\right), \partial D\right)^{n-1} .
$$

(in both (4.6) and (4.7), depends only on $K, n$ and $\delta$.)

ProOF. (4.6) follows directly from (4.5), the following Lemma, and Theorem 3. (4.7) follows directly from (4.6) and (4.3)-(4.5).

Let $\rho$ be the standard hyperbolic metric on $B^{n}$.

LeMmA 2 ([AG2]). Let $f: B^{n} \rightarrow D$ be $K$-quasiconformal. Then $a_{f}(x)$ is quasihyperbolically constant on $B^{n}$, i.e., if $x, y \in B^{n}$ with $\rho(x, y) \leqq M$, then

$$
\frac{a_{f}(x)}{a_{f}(y)} \leqq C,
$$

where $C$ depends only on $M, K$, and $n$.

We will also need the following result:

Lemma 3. Suppose that $f: B^{n} \rightarrow D$ is K-quasiconformal. For each $x \in B^{n}$ define $I_{x}=B(x, 2(1-|x|)) \cap \partial B^{n}$. Then for all $x \in B^{n}$

$$
d(f(x), \partial D) \leqq C_{1} d\left(f\left(I_{x}\right)\right) .
$$

Moreover, if $D$ is a b-John domain, then

$$
d\left(f\left(I_{x}\right)\right) \leqq C_{2} d(f(x), \partial D) .
$$

$\left(C_{1}\right.$ depends only on $K$ and $n, C_{2}$ depends only on $K, n$, and $\left.b.\right)$

The first half of Lemma 3 can be proved using a standard modulus argument [H2, p. 109]. The second half can also be found in [H2]. Actually (4.8) characterizes John domains which are images of quasiconformal maps, i.e. if $f: B^{n} \rightarrow D$ is $K$-quasiconformal and (4.8) is satisfied, then $D$ is a John domain. (See Theorem 3.1 in [H2].)

Our final lemma is due to Gehring [G2, p. 383]:

Lemma 4. Suppose that $f: B^{n} \rightarrow D$ is $K$-quasiconformal. Let $x, y \in B^{n}$ with $\rho(x, y) \leqq M<\infty$. Then $|f(x)-f(y)| \leqq C d(f(x), \partial D)$, where $C$ depends only on $K$, $M$, and $n$. 


\section{Proofs.}

\section{Proof of Theorem A.}

Assume that $D$ is a Jordan domain. We first prove that $a_{f} \in H^{1}$ implies that $\partial \mathrm{D}$ is rectifiable. Assume that $a_{f} \in H^{1}$ and let $\left\{y_{0}, y_{1}, \ldots, y_{n}=y_{0}\right\}$ be an ordered set of points on $\partial B^{2}$. It suffices to show that

$$
\sum_{i=0}^{n-1}\left|f\left(y_{i+1}\right)-f\left(y_{i}\right)\right| \leqq C .
$$

An elementary argument shows that for fixed $\delta, 0<\delta<1$ we can choose $r$ arbitrarily close to 1 and a $\delta$-hyperbolic partition $\left\{x_{1}, x_{2}, \ldots, x_{m}\right\}$ of $\{|x|=r\}$ so that

$$
\left\{r y_{1}, r y_{2}, \ldots, r y_{n}\right\} \subset\left\{x_{1}, x_{2}, \ldots, x_{m}\right\} .
$$

Since $\left|x_{j+1}-x_{j}\right| \sim 1-r$, it follows from Lemma 4 that

$$
\left|f\left(x_{j+1}\right)-f\left(x_{j}\right)\right| \leqq C d\left(f\left(x_{j}\right), \partial D\right) .
$$

Combining this inequality with (5.2) and Lemma 1, we get

$$
\sum_{i=0}^{n-1}\left|f\left(r y_{i+1}\right)-f\left(r y_{i}\right)\right| \leqq C_{1} \int_{0}^{2 \pi} a_{f}\left(r e^{i \theta}\right) d \theta \leqq C_{2} .
$$

(5.1) now follows by taking $r \rightarrow 1$ and using the continuity of $f$ on $\overline{B^{2}}$, (which follows from the fact that $D$ is a Jordan domain [VI, Theorem 17.20]).

We next show that rectifiability of $\partial D$ implies that $a_{f} \in H^{1}$. Assume that $L=\mathscr{H}_{1}(\partial D)<\infty$. Let $0<r<1$ and choose $\left\{y_{0}, y_{1}, \ldots, y_{n}=y_{0}\right\}$ an ordered set of points on $\partial B^{2}$ so that $\left\{r y_{1}, r y_{2}, \ldots, r y_{n}\right\}$ forms a hyperbolic partition of $S_{r}$. Let $I_{j}=\left[y_{j-1}, y_{j}\right]=\left\{e^{i \theta}: \arg \left(y_{j-1}\right) \leqq \theta \leqq \arg \left(y_{j}\right)\right\} \quad$ for $j=1,2, \ldots, n$. Since $\left|y_{j-1}-y_{j}\right| \sim 1-r$, it follows from Lemmas 3 and 4 that

$$
d\left(f\left(r y_{j}\right), \partial D\right) \leqq C d\left(f\left(I_{j}\right)\right)
$$

where $C$ depends only on $K$ and $n$. Now using Lemma 1 and (5.3), we get

$$
\begin{aligned}
\int_{0}^{2 \pi} a_{f}\left(r e^{i \theta}\right) d \theta & \leqq C_{1} \sum_{j=1}^{n} d\left(f\left(r y_{j}\right), \partial D\right) \\
& \leqq C_{2} \sum_{j=1}^{n} d\left(f\left(I_{j}\right)\right) \\
& \leqq C_{2} \sum_{j=1}^{n} \mathscr{H}_{1}\left(f\left(I_{j}\right)\right) \\
& =C_{2} L
\end{aligned}
$$


and the proof of Theorem A is complete.

Proof of Theorem B.

Assume first that $D$ is a Jordan John domain and that $a_{f} \in H^{n-1}$. Let $\varepsilon>0$. By the uniform continuity of $f$ on $\overline{B^{n}}$ ([VI, Theorem 17.20]) we can choose $0<r<1$ and a hyperbolic partition $\left\{x_{1}, x_{2}, \ldots, x_{m}\right\}$ of $S_{r}$ so that

$$
\partial B^{n} \subset \cup_{i} I_{x_{i}}
$$

and

$$
\max _{i} d\left(f\left(I_{x_{i}}\right)\right)<\frac{\varepsilon}{2} .
$$

Now for each $i$ choose a ball $B_{i} \supset f\left(I_{x_{i}}\right)$ satisfying:

$$
d\left(B_{i}\right) \leqq 2 d\left(f\left(I_{x_{i}}\right)\right) .
$$

It then follows from (5.4) that

$$
\partial D \subset \cup_{i} B_{i}
$$

and from (5.6), Lemma 1, and Lemma 3 we get

$$
\begin{aligned}
\sum_{i=1}^{m}\left(d\left(B_{i}\right)\right)^{n-1} & \leqq 2^{n-1} \sum_{i=1}^{m} d\left(f\left(I_{x_{i}}\right)\right)^{n-1} \\
& \leqq C_{1} \sum_{i=1}^{m} d\left(f\left(x_{i}\right), \partial D\right)^{n-1} \\
& \leqq C_{2} \int_{S_{r}}\left(a_{f}(\omega)\right)^{n-1} d \mathscr{H}_{n-1}(\omega) \\
& \leqq C_{3} .
\end{aligned}
$$

Since $d\left(B_{i}\right)<\varepsilon$ by (5.5) and (5.6), it follows that $\mathscr{H}_{n-1}(\partial D) \leqq C_{3}$.

Assume now that $\partial D$ is rectifiable so $\mathscr{H}_{n-1}(\partial D)=M<\infty$. Let $0<r<1$ and choose $\left\{x_{1}, x_{2}, \ldots, x_{m}\right\}$ to be a hyperbolic partition of $S_{r}$. Then from Lemma 1 we have

$$
\int_{S_{r}} a_{f}(\omega)^{n-1} d \mathscr{H}_{n-1}(\omega) \leqq C_{1} \sum_{i=1}^{m} d\left(f\left(x_{i}\right), \partial D\right)^{n-1} .
$$

The second half of Theorem B would now follow from (5.7) if we could prove the following:

Conjecture. Let $f: B^{n} \rightarrow D$ be $K$-quasiconformal with $D$ a Jordan domain. Then for every $x \in B^{n}$ 


$$
\left(d(f(x), \partial D)^{n-1} \leqq C \mathscr{H}_{n-1}\left(f\left(I_{x}\right)\right),\right.
$$

where $C$ is a constant depending only on $K$ and $n$.

Note that if the Conjecture were true, we would actually have the second half of Theorem B without any extra restrictions on $D$. However, so far I have only been able to prove the Conjecture for certain special cases. One such special case is when $D$ is a uniform domain. To prove the Conjecture in this case we need some more notation and a result of Väisälä which is a generalization of a deep theorem of Gehring.

Suppose that $\Gamma$ is a family of curves on $R^{n}$. For each $p$ and $q$ in $(0, \infty)$ we define the $p$-dimensional $q$-modulus of $\Gamma$ by

$$
M_{q}^{p}(\Gamma)=\inf \int_{R^{n}} h^{q} d \mathscr{H}_{p}
$$

where the infimum is taken over all Borel functions $h: R^{n} \rightarrow[0, \infty]$ such that

$$
\int_{\gamma} h d s \geqq 1
$$

for all locally rectifiable cures $\gamma \in \Gamma$. Then Väisälä has proved the following [V3, Theorem 5.6]:

THEOREM 4. Suppose that $2 \leqq p \leqq n, G$ is an open subset of $R^{p}, H>1$, $g: G \rightarrow P \subset R^{n}$ is $H$-quasisymmetric, and $\mathscr{H}_{p}(g(E))<\infty$ for every compact $E \subset G$. Then

$$
M_{p}^{p}(\Gamma) \leqq C M_{p}^{p}(g(\Gamma))
$$

for every path family $\Gamma$ in $G$, where $C$ depends only on $H$ and $n$.

We first note that Theorem 4 obviously remains true if we assume that $G$ is an open subset of $S^{p}=\left\{x \in R^{p+1}:|x|=1\right\}$ rather than of $R^{p}$. We now wish to apply Theorem 4 to prove the Conjecture under the added assumption that $D$ is a uniform domain. Before beginning our proof we note that a simple Moebius transformation argument shows that in place of (5.8) it suffices to show that

$$
(d(f(0), \partial D))^{n-1} \leqq C \mathscr{H}_{n-1}\left(f\left(S^{+}\right)\right)
$$

where $S^{+}$is the upper half sphere

$$
\left\{\left(x_{1}, x_{2}, \ldots, x_{n}\right) \in \partial B^{n}: x_{n} \geqq 0\right\} .
$$

In order to apply Theorem 4 we first note that since $D$ is a uniform John domain (and hence bounded) and $f$ is $K$-quasiconformal, it follows that $f$ is $H$-quasisymmetric on $\partial B^{n}$ (where $H$ depends on $f$ and $D$ ) [V2, Theorem 5.6]. We 
then apply Theorem 4 by taking $G$ to be the interior of $S^{+}, p=n-1$, and $\Gamma$ to be the family of curves in $S^{+}$joining $E_{1}$ to $E_{2}$ where

$$
\begin{gathered}
E_{1}=\left\{\left(x_{1}, x_{2}, \ldots, x_{n}\right) \in S^{+}: x_{n}=0, x_{2} \geqq\left|x_{1}\right|\right\} \\
E_{2}=\left\{\left(x_{1}, x_{2}, \ldots, x_{n}\right) \in S^{+}: x_{n}=0, x_{2} \leqq-\left|x_{1}\right|\right\} .
\end{gathered}
$$

Clearly,

$$
M_{n-1}^{n-1}(\Gamma)=C(n)>0 .
$$

Let $\gamma \in \Gamma$. Then $d(\gamma) \geqq 1$ and we claim that

$$
l(f(\gamma)) \geqq d(f(\gamma)) \geqq C d(f(0), \partial D)>0,
$$

where $C$ depends only on $K$ and $n$.

We use a standard modulus argument to establish the claim. Let $\Theta$ be the family of paths connecting $B(0, r)$ to $\gamma$ in $B^{n}$. Since $d(\gamma) \geqq 1$, we clearly have

$$
M_{n}^{n}(\Theta) \geqq C(r, n)>0 .
$$

By the distortion theorem for quasiconformal mappings [VI, Theorem 18.1] there exists $r=r(K, n)<1$ such that

$$
f(B(0, r)) \subset B\left(f(0), \frac{1}{2} d(f(0), \partial D)\right) .
$$

Fix $0<r<1$ satisfying (5.15). Then standard modulus estimates show that

$$
M_{n}^{n}(f(\Theta)) \rightarrow 0 \quad \text { as } \quad \frac{d(f(\gamma))}{d(f(0), \partial D)} \rightarrow 0 .
$$

Since $M_{n}^{n}(f(\Theta)) \geqq \frac{1}{K} M_{n}^{n}(\Theta)$, the claim now follows from (5.14).

Now define

$$
h(x)= \begin{cases}\frac{1}{C d(f(0), \partial D)} & \text { if } \quad x \in f\left(S^{+}\right) \\ 0 & \text { otherwise. }\end{cases}
$$

Then by (5.13) we have

$$
\int_{f(\gamma)} h d s \geqq 1 \text { for all } \gamma \in \Gamma
$$

and hence by (5.9) and (5.16), 


$$
\begin{aligned}
M_{n-1}^{n-1}(f(\Gamma)) & \leqq \int_{f\left(S^{+}\right)} h^{n-1} d \mathscr{H}^{n-1} \\
& =\left(\frac{1}{C d(f(0), \partial D)}\right)^{n-1} \mathscr{H}_{n-1}\left(f\left(S^{+}\right)\right) .
\end{aligned}
$$

Combining (5.17), (5.10) with $p=n-1$, and (5.12) now gives us the Conjecture (with the added assumption that $D$ is a uniform domain and with $C$ dependent on $f, D$ and $n$ ). This completes the proof of the converse part of Theorem B.

\section{Proof of Theorem D.}

Assume that $D \subset R^{2}$ is a Jordan domain and that $a_{f}^{*} \in L^{1}$. Define

$$
h(t)=f\left(e^{i t}\right) \text { for } 0 \leqq t \leqq 2 \pi .
$$

Let $\left[a_{1} b_{1}\right],\left[a_{2}, b_{2}\right], \ldots,\left[a_{n}, b_{n}\right]$ be a pairwise disjoint collection of subintervals of $[0,2 \pi]$. Fix $j, 1 \leqq j \leqq n$ and let $0<r<1$. Let $\left\{x_{1}, x_{2}, \ldots, x_{m}\right\}$ be a hyperbolic partition of $\left\{x \in S_{r}: a_{j} \leqq \arg \left(\frac{x}{|x|}\right) \leqq b_{j}\right\}$ with $x_{1}=r e^{i a_{j}}$ and $x_{m}=r e^{i b_{j}}$. Then applying Lemma 1 and Lemma 4, we get

$$
\begin{aligned}
\left|f\left(x_{1}\right)-f\left(x_{m}\right)\right| & \leqq \sum_{j=1}^{m-1}\left|f\left(x_{j}\right)-f\left(x_{j+1}\right)\right| \\
& \leqq C_{1} \sum_{j=1}^{m-1} d\left(f\left(x_{j}, \partial D\right)\right) \\
& \leqq C_{2} \int_{a_{j}}^{b_{j}} a_{f}\left(r e^{i \theta}\right) d \theta \\
& \leqq C_{2} \int_{a_{j}}^{b_{j}} a_{f}^{*}\left(e^{i \theta}\right) d \theta
\end{aligned}
$$

Now letting $r \rightarrow 1$, summing over $j$, and using the continuity of $f$ on $\overline{B^{2}}$, we get

$$
\sum_{j=1}^{n}\left|h\left(b_{j}\right)-h\left(a_{j}\right)\right| \leqq C \sum_{j=1}^{n} \int_{a_{j}}^{b_{j}} a_{f}^{*}\left(e^{i \theta}\right) d \theta
$$

Since $a_{f}^{*} \in L^{1}$, it follows that $f$ is absolutely continuous on $\partial B^{2}$ as desired.

Before beginning the proof of Theorem $\mathrm{E}$ we define the maximal function and recall some basic facts about it. Let $g: R \rightarrow R$ be a locally integrable function. The maximal function of $g$ is defined by

$$
M g(x)=\sup _{I} \frac{1}{|I|} \int_{I}|g(t)| d t
$$


where the sup is taken over all intervals $I$ which contain $x$. We next define

$$
\begin{aligned}
& g \in L \log L([0,1])=L \log L \text { if } \\
& \int_{[0,1]}|g(x)| \log (2+|g(x)|)<\infty .
\end{aligned}
$$

We will need the following fundamental result about the maximal function for the proof of Theorem $\mathrm{E}[\mathrm{S}]$ :

Proposition 1. Let $g \geqq 0$ be integrable on $[0,1]$. Then $M g \in L^{1}[0,1]$ if and only if $g \in L \log L$.

Proof of Theorem E.

We start with a definition. We say that $x \in B^{2}$ is hyperbolically related to the interval $I$ if $|x|=1-|I|$ and $\arg \left(\frac{x}{|x|}\right)=\operatorname{midpoint}(I)$. Let $f: B^{2} \rightarrow B^{2}$ be $K$-quasiconformal with $f(1)=1$ and define $g:[0,2 \pi) \rightarrow \partial B^{2}$ by $g(t)=e^{i t}$. Let $h=g^{-1} \circ f \circ g$ so $h$ is a homeomorphism of $[0,2 \pi)$ onto itself. We extend $h$ recursively by $h(t+2 \pi)=h(t)+2 \pi$ so $h$ is a homeomorphism of $R$ onto $R$. Let $0 \leqq a<b \leqq 2 \pi$. Then by Theorem 3, Lemma 3, the definition of $h$, and the fact that $B^{2}$ is a John domain, it follows that

$$
a_{f}(x) \sim \frac{h(b)-h(a)}{b-a},
$$

whenever $x$ is hyperbolically related to $[a, b]$. Here $\sim$ depends only on $K$. If we assume that $h$ is absolutely continuous, it follows from (5.20) and Lemma 2 that

$$
a_{f}^{*}(t) \sim M h^{\prime}(t) \text { a.e. in }[0,2 \pi]
$$

where $\sim$ again depends only on $K$. Since $h^{\prime} \geqq 0$ a.e., it follows from (5.21) and Proposition 1 that $a_{f}^{*} \in L^{1}[0,2 \pi]$ if and only if $h^{\prime} \in L \log L([0,2 \pi])$. Hence, to prove Theorem $\mathrm{F}$ it suffices to construct an $f$ such that for the corresponding $h$ we have $h^{\prime} \notin L \log L([0,2 \pi])$. By Beurling-Ahlfors' result [BA, Theorem 1], this is equivalent to constructing a homeomorphism $h:[0,1] \rightarrow[0,1]$ which is quasisymmetric and absolutely continuous but such that $h^{\prime} \notin L \log L$.

We construct $h$ by modifying an example of Tukia [T1]. The functions $h_{n}$ which we will define were used by him in a limiting procedure to construct a quasisymmetric homeomorphism from $R$ to $R$ which does not preserve Hausdorff dimension. We borrow his notation and approach in defining $h_{n}$.

For $n \geqq 0$ and $0 \leqq j<2^{n}$ we define intervals $I_{n, j}$ inductively as follows: Let $I_{0,0}=[0,1]$. In general, given the interval $I_{n, j}$, we divide it into a left subinterval $I_{n+1,2 j}$ and a right subinterval $I_{n+1,2 j+1}$ in the following proportions: 


$$
\begin{gathered}
\frac{\left|I_{n+1,2 j}\right|}{\left|I_{n, j}\right|}=\left\{\begin{array}{l}
\frac{2}{3} \text { if } j \text { is even } \\
\frac{1}{3} \text { if } j \text { is odd }
\end{array}\right. \\
\frac{\left|I_{n+1,2 j+1}\right|}{\left|I_{n, j}\right|}= \begin{cases}\frac{1}{3} \text { if } j \text { is even } \\
\frac{2}{3} \text { if } j \text { is odd. }\end{cases}
\end{gathered}
$$

Now for $n \geqq 0$ and $0 \leqq j<2^{n}$ we define

$$
J_{n, j}=\left[\frac{j}{2^{n}}, \frac{j+1}{2^{n}}\right] .
$$

For fixed $n \geqq 0$ we now define $h_{n}$ to be the homeomorphism of $[0,1]$ which is linear on each $J_{n, j}$ and such that

$$
h_{n}\left(J_{n, j}\right)=I_{n, j} \text { for } 0 \leqq j<2^{n} .
$$

Making use of the following proposition [T1], it then follows that there exists $K<\infty$ such that

$$
h_{n} \text { is } K \text {-quasisymmetric for all } n \text {. }
$$

Proposition 2. Let $h$ be an increasing homeomorphism of $[0,1]$ and define

$$
K_{n, j}=\frac{h\left(\frac{j+1}{2^{n}}\right)-h\left(\frac{j}{2^{n}}\right)}{h\left(\frac{j}{2^{n}}\right)-h\left(\frac{j-1}{2^{n}}\right)} \quad n>0 \quad 0<j<2^{n} .
$$

Then $h$ is quasisymmetric if and only if the $K_{n, j}$ 's are bounded away from 0 and $\infty$.

We next define homeomorphisms

$$
\begin{aligned}
& h_{n}(x) \quad 0 \leqq x \leqq \frac{2}{2^{n}} \\
& p_{n}(x)=h_{n-1}(x) \quad \frac{2}{2^{n}} \leqq x \leqq \frac{4}{2^{n}} \\
& h_{1}(x) \quad \frac{2^{n-1}}{2^{n}} \leqq x \leqq \frac{2^{n}}{2^{n}} \\
& h_{1}(x) \quad 0 \leqq x \leqq \frac{1}{2} \\
& h_{2}(x) \quad \frac{1}{2} \leqq x \leqq \frac{3}{4} \\
& q_{n}(x)=\quad \vdots \\
& h_{n}(x) \quad \frac{2^{n}-2}{2^{n}} \leqq x \leqq \frac{2^{n}}{2^{n}} .
\end{aligned}
$$


We then extend $h_{n}$ to a homeomorphism $h_{n}^{*}$ of $[-2,3]$ by defining

$$
h_{n}^{*}(x)=\left\{\begin{array}{lr}
x & -2 \leqq x \leqq-1 \\
-p_{n}(-x) & -1 \leqq x \leqq 0 \\
h_{n}(x) & 0 \leqq x \leqq 1 \\
2-q_{n}(2-x) & 1 \leqq x \leqq 2 \\
x & 2 \leqq x \leqq 3 .
\end{array}\right.
$$

Finally, we renormalize so that we again have a homeomorphism of $[0,1]$ by defining

$$
k_{n}(x)=\frac{1}{5}\left(h_{n}^{*}(5 x-2)+2\right) .
$$

Using Proposition 2, it is an easy, but tedious, task to verify that

$$
k_{n} \text { is } K^{\prime} \text { - quasisymmetric on }[0,1],
$$

where $K^{\prime}$ depends only on $K$. This is, in fact, why the auxiliary mappings $p_{n}$ and $q_{n}$ were introduced. Note also that

$$
k_{n}(x)=x \quad \text { for } \quad x \in\left[0, \frac{1}{5}\right] \cup\left[\frac{4}{5}, 1\right] .
$$

Now let $\left[a_{1}, b_{1}\right],\left[a_{2}, b_{2}\right], \ldots$ be a sequence of pairwise disjoint subintervals of $[0,1]$ and $m_{1}, m_{2}, \ldots$ a sequence of positive integers. Define $k$ on $[0,1]$ as follows:

$$
k(x)= \begin{cases}x & \text { if } x \in[0,1] \backslash\left\{\cup\left[a_{n}, b_{n}\right]\right\} \\ \left(b_{n}-a_{n}\right) k_{m_{n}}\left(\frac{x-a_{n}}{b_{n}-a_{n}}\right)+a_{n} & \text { if } a_{n} \leqq x \leqq b_{n} .\end{cases}
$$

Then $k$ is clearly an absolutely continuous homeomorphism of $[0,1]$ and again a simple, but tedious, argument using (5.24), (5.25) and Proposition 2 shows that $k$ is $K^{\prime \prime}$-quasisymmetric, where $K^{\prime \prime}$ depends only on $K$.

It remains to show that we can choose $\left[a_{n}, b_{n}\right]$ and $m_{n}$ so that $k^{\prime} \notin L \log L$. First of all a simple calculation using (5.22) shows that

$$
\begin{aligned}
& \int_{0}^{1} h_{n}^{\prime}(x) \log \left(2+h_{n}^{\prime}(x)\right) d x= \\
& \sum_{j=0}^{n}\left(\begin{array}{l}
n \\
j
\end{array}\right)\left(\frac{2}{3}\right)^{j}\left(\frac{1}{3}\right)^{n-j} \log \left[2+\left(\frac{4}{3}\right)^{j}\left(\frac{2}{3}\right)^{n-j}\right]
\end{aligned}
$$

We now estimate the right hand side of (5.27). Note that $\left(\frac{4}{3}\right)^{a}\left(\frac{2}{3}\right)^{1-a}=1$ if $a=\log _{2}\left(\frac{3}{2}\right)<\frac{2}{3}$. Choose $b: a<b<\frac{2}{3}$. Then 


$$
\begin{aligned}
& \sum_{j=0}^{n}\left(\begin{array}{l}
n \\
j
\end{array}\right)\left(\frac{2}{3}\right)^{j}\left(\frac{1}{3}\right)^{n-j} \log \left[2+\left(\frac{4}{3}\right)^{j}\left(\frac{2}{3}\right)^{n-j}\right] \\
& \geqq \sum_{b n \leqq j \leqq n}\left(\begin{array}{l}
n \\
j
\end{array}\right)\left(\frac{2}{3}\right)^{j}\left(\frac{1}{3}\right)^{n-j} \log \left[2+\left(\frac{4}{3}\right)^{j-a n}\left(\frac{4}{3}\right)^{a n}\left(\frac{2}{3}\right)^{n-j}\right] \\
& \geqq \sum_{b n \leqq j \leqq n}\left(\begin{array}{l}
n \\
j
\end{array}\right)\left(\frac{2}{3}\right)^{j}\left(\frac{1}{3}\right)^{n-j} \log \left[2+\left(\frac{4}{3}\right)^{(b-a) n}\left(\frac{4}{3}\right)^{a n}\left(\frac{2}{3}\right)^{(1-a) n}\right] \\
& \geqq(b-a) n \log \left(\frac{4}{3}\right) \sum_{b n \leqq j \leqq n}\left(\begin{array}{l}
n \\
j
\end{array}\right)\left(\frac{2}{3}\right)^{j}\left(\frac{1}{3}\right)^{n-j} .
\end{aligned}
$$

Since $b<\frac{2}{3}$, it follows that for $n$ large enough

$$
\sum_{b n \leqq j \leqq n}\left(\begin{array}{l}
n \\
j
\end{array}\right)\left(\frac{2}{3}\right)^{j}\left(\frac{1}{3}\right)^{n-j} \geqq \frac{1}{2} .
$$

Hence the righthand side of (5.27) approaches $\infty$ as $n \rightarrow \infty$. Hence, it follows from the definition of $k_{n}$ that

$$
\int_{0}^{1} k_{n}^{\prime} \log \left(2+k_{n}^{\prime}\right)=I_{n} \rightarrow \infty \text { as } n \rightarrow \infty .
$$

Then from (5.26) we get

$$
\int_{0}^{1} k^{\prime}(x) \log \left(2+k^{\prime}(x)\right) \geqq \sum\left(b_{n}-a_{n}\right) I_{m_{n}} .
$$

Hence we only need to choose $a_{n}, b_{n}$ and $m_{n}$ so that

$$
\sum\left(b_{n}-a_{n}\right) I_{m_{n}}=\infty
$$

and we are done with the proof.

Proof of Theorem F.

Assume that

$$
\sup _{r}\left(\int_{0}^{2 \pi} a_{f}\left(r e^{i \theta}\right)^{p} d \theta\right)=L<\infty
$$

for some $p>1$. Define

$$
h_{r}(t)=f\left(r e^{2 \pi i t}\right) \text { for } 0 \leqq t \leqq 1 \text { and } 0 \leqq r \leqq 1 .
$$

Let $\varepsilon>0$. Suppose $M, N$ are positive integers and

$$
0 \leqq m_{1}<m_{2}<\ldots<m_{M} \leqq N-1 .
$$

Then it suffices to show that we can choose $\delta>0$ such that if $\frac{M}{N}<\delta$, then 


$$
\sum_{j=1}^{M}\left|h_{1}\left(\frac{1+m_{j}}{N}\right)-h_{1}\left(\frac{m_{j}}{N}\right)\right|<\varepsilon
$$

Let $r=1-\frac{1}{N}$ and $\theta_{j}=\frac{2 \pi m_{j}}{N}$ for $j=1,2, \ldots M$. Then Lemma 4, Lemma 1 and the definition of $h$ yield

$$
\sum_{j=1}^{M}\left|h_{r}\left(\frac{1+m_{j}}{N}\right)-h_{r}\left(\frac{m_{j}}{N}\right)\right| \leqq C \sum_{j=1}^{M} \int_{\theta_{j}}^{\theta_{j}+\frac{2 \pi}{N}} a_{f}\left(r e^{i \theta}\right) d \theta .
$$

Now applying Jensen's inequality twice we get

$$
\begin{aligned}
\left(\sum_{j=1}^{N}\left|h_{r}\left(\frac{1+m_{j}}{N}\right)-h_{r}\left(\frac{m_{j}}{N}\right)\right|\right)^{p} & \leqq C_{1} M^{p-1} \sum_{j=1}^{M}\left(\int_{\theta_{j}}^{\theta_{j}+\frac{2 \pi}{N}} a_{f}\left(r e^{i \theta}\right) d \theta\right)^{p} \\
& \leqq C_{1} M^{p-1} \sum_{n=0}^{N-1}\left(\int_{\frac{2 \pi n}{N}}^{\frac{2 \pi(n+1)}{N}} a_{f}\left(r e^{i \theta}\right) d \theta\right)^{p} \\
& \leqq C_{1} M^{p-1}\left(\frac{2 \pi}{N}\right)^{p-1} \sum_{n=0}^{N-1} \int_{\frac{2 \pi n}{N}}^{\frac{2 \pi(n+1)}{N}} a_{f}\left(r e^{i \theta}\right)^{p} d \theta \\
& \leqq C_{2}\left(\frac{M}{N}\right)^{p-1} L .
\end{aligned}
$$

Taking the limit as $r \rightarrow 1$ completes the proof.

ACKNOWLEDGEMENT. The research for this paper was done while the author was visiting at the University of Uppsala in Sweden. He would like to thank the university for its hospitality and generous support.

\section{REFERENCES}

AG1. K. Astala, F. W. Gehring, Quasiconformal analogues of theorems of Koebe and Hardy-Littlewood, Mich. Math. J. 32 (1985), 99-107.

AG2. K. Astala, F. W. Gehring, Injectivity, the BMO Norm and the Universal Teichmüller Space, J. Analyse Math. 46 (1986), 16-56.

BA. A. Beurling, L. Ahlfors, The boundary correspondence under quasiconformal mappings, Acta Math. 96 (1956), 125-142.

C. L. Carleson, On mappings, conformal at the boundary, J. Analyse Math. 19 (1967), 1-13.

G1. F. W. Gehring, Lower dimensional absolute continuity properties of quasiconformal mappings, Math. Proc. Cambridge Philos. Soc. 78 (1975), 81-93.

G2. F. G. Gehring, Rings and quasiconformal mappings in space, Trans. Amer. Math. Soc. 103 (1962), 353-393.

H1. J. Heinonen, On the boundary absolute continuity of quasiconformal mappings, Amer. J. Math. 116 (1994), 1-23. 
H2. J. Heinonen, Quasiconformal mappings onto John domains, Rev. Mat. Iberoamericama 5 (1989), 97-123.

H3. J. Heinonen, Unpublished notes.

S. E. M. Stein, Note on the class $L \log L$, Studia Math. 32 (1969), 305-310.

T1. P. Tukia, Hausdorff dimension and quasisymmetric mappings, Math. Scand. 65(1989), 152-160.

V1. J. Väisälä, Lectures on n-dimensional Quasiconformal Mappings, Springer-Verlag, New York, 1971.

V2. J. Väisälä, Quasimöbius maps, J. Analyse Math. 44 (1984/85), 218-234.

V3. J. Väisälä, Quasisymmetric Embeddings in Euclidean Spaces, Trans. Amer. Math. Soc. 264 (1981), 191-204.

ST. OLAF COLLEGE

NORTHFIELD, MN 55057

U.S.A. 